\title{
Design and Rationale of Health-Related Quality of Life and Patient-Reported Outcomes Assessment in the Frequent Hemodialysis Network Trials
}

\author{
Manisha Jhamb ${ }^{b}$ Manjula K. Tamurad Jennifer Gassman ${ }^{\mathrm{e}}$ \\ Amit X. Garg ${ }^{a}$ Robert M. Lindsay ${ }^{a}$ Rita S. Suri ${ }^{a}$ George Ting ${ }^{f}$ \\ Fredric O. Finkelstein ${ }^{g}$ Scott Beach ${ }^{c}$ Paul L. Kimmel ${ }^{\text {h }}$ Mark Unruh $^{b}$ \\ for the Frequent Hemodialysis Network Trial Group \\ ${ }^{a}$ Division of Nephrology, University of Western Ontario, London, Ont., Canada; ${ }^{b}$ Division of Nephrology, \\ University of Pittsburgh Medical Center, ' University Center for Social and Urban Research, University of Pittsburgh, \\ Pittsburgh, Pa., ${ }^{d}$ Division of Nephrology, Stanford University School of Medicine, Palo Alto, Calif., ${ }^{e}$ Department of \\ Quantitative Health Sciences, Cleveland Clinic Foundation, Cleveland, Ohio, ${ }^{\mathrm{f}}$ El Camino Hospital, Mountain View, \\ Calif., ${ }^{9}$ Department of Medicine, Hospital of St. Raphael, Yale University School of Medicine, New Haven, Conn., \\ and hivision of Kidney Urologic and Hematologic Diseases, National Institute of Diabetes and Digestive and \\ Kidney Diseases, National Institutes of Health, Bethesda, Md., USA
}

\section{Key Words}

Health-related quality of life • Frequent Hemodialysis

Network - End-stage renal disease

\begin{abstract}
Background: End-stage renal disease patients experience significant impairments in health-related quality of life (HRQOL). Testing various strategies to improve patient HRQOL in multicenter clinical trials, such as the Frequent Hemodialysis Network (FHN) trials is vitally important. Aims: The aim of this paper is to describe the design and conduct of HRQOL and patient-reported outcomes (PRO) assessment in the FHN trials. Methods: In the FHN trials, HRQOL was examined as a multidimensional concept, and the SF-36 RAND Physical Health Composite score was one of the co-primary outcomes. The instruments completed to assess HRQOL included the Medical Outcomes Study Short Form SF-36, Health Utilities Index 3, Sleep Problems Index, Beck Depres-
\end{abstract}

sion Inventory and feeling thermometer. These instruments have been shown to have high reliability, validity and responsiveness to change in the end-stage renal disease population. Additional items evaluating PRO including sexual function, time to recovery after dialysis and patients' selfperceived burden to caregiver were also assessed. All questionnaires were administered by trained interviewers using computer-assisted telephone interviewing to ensure blinding and minimizing selection bias. Interim analysis reveals that these instruments can be used to collect a comprehensive set of HRQOL measures with minimal patient burden. Conclusions: Accurate measurement of $\mathrm{HRQOL}$ and $\mathrm{PRO}$ can help us test whether hemodialysis interventions improve the health and well-being of this compromised patient population. We have shown that a comprehensive set of HRQOL measures can be centrally collected through telephone interviews in a blinded fashion, in a way that is well tolerated with minimum respondent burden.

Copyright $\odot 2011$ S. Karger AG, Basel

Mark Unruh, MD, MSc

University of Pittsburgh School of Medicine

200 Lothrop Street, PUH C-1111

Pittsburgh, PA 15213 (USA)

Tel. +1 412647 2571, Fax +1 412647 4787, E-Mail unruh@pitt.edu 


\section{Introduction}

Health-related quality of life (HRQOL) may be an important and often overlooked outcome in patients undergoing maintenance hemodialysis (HD). Patients with chronic kidney disease and end-stage renal disease (ESRD) on maintenance renal replacement therapy (RRT) experience significant physical, emotional, mental and psychosocial impairments which are reflected in poor HRQOL scores [1-3]. Over half of maintenance dialysis patients experience physical and emotional symptoms including fatigue, muscle cramps, pain, difficulty with sleep and sexual dysfunction which are directly correlated with impaired HRQOL and greater levels of depression [4]. The prevalence of fatigue is reported to range from $60 \%$ to as high as $97 \%$ in patients on long-term RRT [3]. While $25-30 \%$ of the maintenance dialysis patients suffer from depression $[5,6]$, poor sleep quality has also been widely noted [7]. In these patients on maintenance $\mathrm{RRT}$, it is both the disease and the treatment which contribute to poor HRQOL and patient-reported outcomes (PROs). A PRO is any report of the status of patient's health condition that comes directly from the patient, without interpretation of the patient's response by a clinician or anyone else. PRO is a broad term that includes any data collected by self-report from the patients such as HRQOL, symptoms, and health utilities.

HRQOL is an outcome that is directly important to patients, unlike outcomes such as physiologic or biochemical measures that do not directly influence how patients feel [8]. Patients undergoing maintenance dialysis give more importance to improved quality of life than survival. In a study of 100 maintenance HD patients, $94 \%$ and $57 \%$ of the patients would undergo more frequent dialysis if it improved their energy level and sleep quality, respectively, but only $19 \%$ would undergo this for increase in survival of up to 3 years [9]. Furthermore, HRQOL has been shown to be an independent predictor of survival in ESRD patients [10, 11]. Dialysis Outcomes and Practice Patterns Study, an international, prospective, observational study of randomly selected HD patients found that lower HRQOL scores were strongly associated with higher risk of death and hospitalization in HD patients, independent of a series of demographic and comorbid factors [12]. Similarly, in a large cross-sectional study among approximately 14,000 prevalent dialysis patients, HRQOL measures of Physical Component Summary (PCS) and Mental Component Summary (MCS) were consistent predictors of hospitalizations and mortality rates even after adjustment for clinically relevant factors [11].
Insofar as HRQOL and PRO measure patient well-being, improving HRQOL is an important goal of RRT. The value of HRQOL measurement to improve clinical care and as a legitimate outcome of clinical trials has been recognized by many in the research community [ $[13$, 14].Concurrent measurement of several dimensions of HRQOL and variables that influence HRQOL is crucial [15]. However, which domains best represent patient wellbeing, and which are the best HRQOL instruments remain unresolved questions. The Frequent Hemodialysis Network (FHN) Trials are testing the hypothesis that more frequent HD improves the health and well-being of dialysis patients [16]. The FHN trials are two separate randomized controlled trials. In the Nocturnal Trial, 5-6 nights weekly HD was compared to thrice weekly HD. In the Daily Trial, short-frequent in-center HD was compared to thrice weekly in-center HD. The two co-primary outcomes of the study were: (1) a composite of mortality with the change in the 36-Item Short-Form Health Survey (SF-36) RAND Physical Health Composite (PHC), and (2) a composite of mortality and change in left ventricular mass. Both the Nocturnal and Short Daily trials have been collecting long-term follow-up (up to 24 months) of HRQOL.

A valid baseline measure of HRQOL was a prerequisite prior to randomization in the FHN Trials. This was to ensure that baseline data are available for follow-up comparisons in HRQOL. The domains selected by FHN investigators were physical and mental well-being, depressive symptoms, and sleep. The aim of this paper is to describe the design, rationale and conduct of the HRQOL and PRO assessment in the FHN trials (table 1).

\section{Dialysis Clinical Trials to Improve HRQOL in HD Patients}

Given the importance of HRQOL in patients with ESRD, many investigators have studied various modifications of HD to improve HRQOL and clinical outcomes. The HEMO Study examined the effect of HD dose and membrane flux on HRQOL using Kidney Disease Quality of Life-Long Form and Index of Well-Being questionnaires, and found no clinically meaningful benefits of either of these interventions on any of the HRQOL indices [17]. Small observational reports and trials have suggested that more frequent HD treatments may provide more physiologic RRT and improve clinical outcomes and HRQOL [18-20]. In a systematic review of studies on daily HD by Suri et al. [19], significant improvements in SF- 
Table 1. Instruments used to assesses PRO in the FHN

\begin{tabular}{lll}
\hline Domain & Definition & Instrument \\
\hline $\begin{array}{l}\text { Core assessments } \\
\text { Physical functional status }\end{array}$ & $\begin{array}{l}\text { Capacity to perform basic physical activities of daily } \\
\text { life, ratings of vitality and bodily pain } \\
\text { General mood or affect, severity of depression }\end{array}$ & SF-36 \\
$\begin{array}{l}\text { Psychological symptoms } \\
\text { Social/role functioning }\end{array}$ & $\begin{array}{l}\text { Extent to which health interferes with social } \\
\text { activities/responsibilities } \\
\text { General evaluation of health status and well-being }\end{array}$ & SF-36 \\
Global quality of life & SF-36, feeling thermometer \\
\hline $\begin{array}{l}\text { Supplemental assessments } \\
\text { Physical functioning in specific areas } \\
\text { Hemodialysis-specific concerns }\end{array}$ & $\begin{array}{l}\text { Limitations in ambulation, mobility, body care, sleep } \\
\text { Positive and negative effects }\end{array}$ & $\begin{array}{l}\text { HUI3, sleep problems index } \\
\text { Self-perceived burden to caregiver, sexual } \\
\text { function, recovery time, inconvenience }\end{array}$ \\
\hline
\end{tabular}

36 PCS and MCS domains, minutes to recovery after dialysis and health utilities were found, but these results were not consistent across studies. A systematic review of studies evaluating nocturnal $\mathrm{HD}$ revealed highly variable improvement in HRQOL after conversion from conventional to nocturnal HD [20]. For example, some small studies reported greater than $85 \%$ improvement in selected HRQOL domains, while others noted only minimal improvements [21,22]. Cross-sectional comparison of HRQOL, depression and intrusiveness of illness among nocturnal $\mathrm{HD}$ and peritoneal dialysis patients found no significant differences [23]. A 6-month randomized controlled study of conventional versus 6 nights per week nocturnal HD by the Alberta Kidney Disease Network showed significant improvements in selected kidney-specific domains of QOL (effects of kidney disease and burden of kidney disease) but did not improve overall HRQOL as measured by Kidney Disease Quality of Life Short Form and Euroqol EQ-5D. Assessment of HRQOL with extended follow-up of participants after completion of the trial also revealed similar results [24]. In the London Daily/Nocturnal Hemodialysis Study, comparison of 23 patients on short daily or long nocturnal HD with 22 conventional thrice-weekly HD patients showed that quotidian HD patients maintained functionality throughout the study period, whereas control patients showed a significant loss. Given the choice, all patients chose to remain on quotidian HD therapy after switching from conventional HD therapy [25]. More recently, interim analysis from the ongoing FREEDOM (Following Rehabilitation, Economics and Everyday-Dialysis Outcome Measurements) study revealed significant decrease in mean Beck Depression Inventory (BDI) score and postdialysis recovery time over 12 months in patients undergoing daily (6 times per week) HD [26].

\section{Conceptual Model of HRQOL in FHN Trials}

Patient-centered quality of life outcomes can be conceptualized as not merely an absence of disease or infirmity but also the overall well-being of the patient [27, 28]. In the FHN trials, HRQOL was examined with multidimensional assessment (fig. 1). One of two study coprimary outcomes was a composite of physical well-being as measured by an SF-36 RAND summary score (Physical Health Component) and mortality. The FHN trials also measured symptoms of depression, mental health, health utility, sleep problems, self-perceived burden of care and sexual function. These aspects of HRQOL are domains that may be impacted by the study intervention and are valued by patients with ESRD.

\section{Measures to Assess HRQOL in the FHN Trials}

One of the main challenges to improving HRQOL is the accuracy and validity of standardized assessment tools to measure the HRQOL domains. The optimal HRQOL assessment tools in the FHN trials would be multidimensional, incorporate both generic and diseasespecific measures, be easy to administer and yield indices that measure differences that can be clearly compared to normative data. They should have high reliability, valid- 


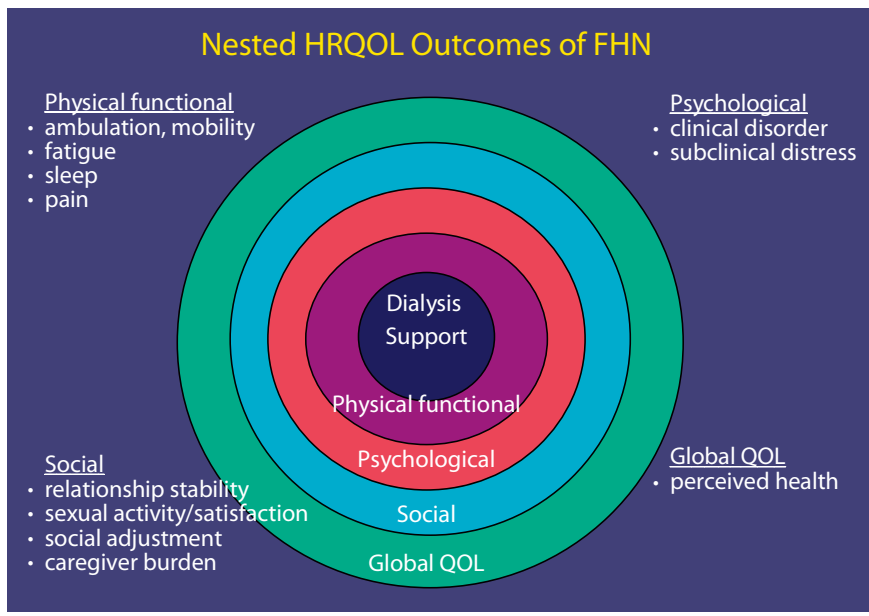

Fig. 1. Multidimensional assessment of HRQOL in FHN.

ity and responsiveness to change. The following measures, which have been tested in the ESRD population, were thought to best meet the above criteria and were used in the FHN trials.

\section{Medical Outcomes Study SF-36}

The four most commonly used generic global HRQOL assessment instruments used in the ESRD population have been the Medical Outcomes Study SF-36 [29, 30], the Sickness Impact Profile (SIP) [31], the Campbell Indices $[32,33]$ and the Nottingham Health Profile (NHP) [34].

The SIP questionnaire, which has 12 categories of health-related activities, has demonstrated high reliability (0.88-0.92) [35] but results of validity testing in the ESRD population have varied [33]. A limitation of SIP is that the 136 items take about $25 \mathrm{~min}$ to complete [36]. For the NHP, there is no evidence of validity testing in the ESRD population [37].

The SF-36 is one of the most commonly used instruments to measure HRQOL in the ESRD population [29, $30,36,37]$. It has two components, (PCS and MCS), and 8 subscales which have been tested extensively for reliability and validity in HD patients [14]. In addition, norms for the general US population have been reported. The survey is well-accepted by HD patients, taking only 5-10 min to complete [38, 39]. SF-36 function score has been shown to be responsive to clinical change on implementing interventions such as the use of erythropoietin to reduce anemia in the ESRD population [40]. It has also been shown to be responsive to clinical change for daily and nocturnal HD [25]. Thus, the RAND PHC from the
SF-36 was used as the global HRQOL assessment tool for the FHN trials. The RAND PHC score, which is based on the same SF-36 scales as the PCS score (physical function, role-physical, pain, general health perceptions), was used as a component of a co-primary outcome rather than one of the SF-36 summary scales (PCS, MCS). This is because the Medical Outcomes Study scoring system makes the assumption that there is no correlation between physical and mental health. In order to support this assumption, some of the subscales have a negative weighting in the PCS (and MCS). For example, in scoring the MCS, the physical functioning score is negatively weighted such that a person with poor physical function can have their MCS scores somewhat increased not because of better mental well-being, but due to poor physical well-being. Thus, the PCS and MCS can in some cases produce incongruous results when respondents score at the extremes of the scales. For example, the MCS failed to detect major clinical differences associated with disease progression, despite significant differences in its component subscales [41]. Unlike the PCS, however, the scoring algorithm used to calculate the PHC is based on nonorthogonal factor rotation. This allows the PHC to correlate with mental health, unlike the PCS.

\section{Health Utilities Index 3}

Utility scores provide an overall assessment of HRQOL of patients and are useful in cost-utility and cost-effectiveness analyses. Changes in quantity of life, as measured in years, can be combined with changes in quality of life, as measured in utilities, to determine the number of quality-adjusted life years gained by a particular health intervention. This can be compared with the incremental cost of the program to determine the cost per qualityadjusted life-year gained. Utilities may be measured directly by means of techniques such as visual analog scaling, standard gamble, or time trade-off (TTO). The TTO is a preference-based approach that provides a health index [36]. Although it has been shown to have adequate reliability and validity [42], it is not very responsive to change with interventions [43]. Utilities may be determined indirectly by means of a preference-weighted multi-attribute health status classification system such as the Health Utilities Index 3 (HUI3) and EuroQOL-5D $[44,45]$.

The HUI3 is a 21-item generic health instrument for determining overall utility associated with particular health states. The HUI questionnaire is composed of eight attributes of high importance to members of the general population: vision, hearing, speech, ambulation, 
dexterity, emotion, cognition, and pain. A preferencebased scoring function, based on multi-attribute utility theory, allows conversion of questionnaire responses into measures of overall health utility, which can then be used to calculate quality-adjusted life years [46]. HUI3 provides comprehensive, reliable, responsive and valid measures of health status and HRQOL [46]. In the London study, the HUI3 showed differential responsiveness to change in nocturnal versus conventional HD patients during longitudinal follow-up. Test-retest reliability at 4 weeks was 0.77 [25]. Given the experience with HUI3 in ESRD patients, it was used for quality-adjustment of survival time in the economic evaluation of the FHN trials.

\section{Feeling Thermometer}

The feeling thermometer is a single question that asks subjects to rate their own health on a visual analog scale from 0 to 100, with 0 being dead and 100 being perfect health $[47,48]$. The feeling thermometer has been used in numerous studies to permit patients to provide preference ratings of their own health status (health utilities). Although it has not been shown to discriminate between dialysis modalities, it has demonstrated responsiveness to therapy in multiple health states $[47,48]$. Thus, the 12 month change in feeling thermometer score was used as an additional secondary outcome in this trial.

\section{Beck Depression Inventory}

Some of the measures that have been used to screen for depression in patients with ESRD are the BDI, Hamilton Rating Scale for Depression (HAMD), Nine-Question Patient Health Questionnaire (PHQ-9), Center for Epidemiologic Studies Depression Scale, and the Cognitive Depression Index (a subset of BDI) [49]. The BDI, a 21-question validated survey presented in multiple choice format, measures the presence and degree of depression in adults. Each of the answers is scored on a 0-3 scale, and inventory items correspond to a specific category of depressive symptom and/or attitude. BDI results are highly correlated with psychiatrists' ratings using the Hamilton Rating Scale (0.75-0.80). Based on a pooled analysis of studies in primary care, the sensitivity and specificity of the BDI in detecting moderate-severe depression are approximately $90 \%$ and $56 \%$, respectively. Depressive symptoms are frequently encountered in patients with ESRD [50]. The BDI has been frequently used to assess presence and severity of depression in patients with ESRD [49, 51]. High scores on the BDI have predictive value at diagnosing depression and are associated with mortality in this patient population $[52,53]$. BDI has also been shown to be responsive over time in ESRD patients [49]. Thus, BDI was chosen to assess depression in the FHN study.

\section{The Medical Outcomes Study Sleep Problems Index}

Sleep is an important aspect of HRQOL in patients with ESRD [9]. While there are a large number of potential sleep quality instruments [54], a limited number have been used in ESRD patients. The Epworth Sleepiness Scale (ESS) is an 8 -item measure of sleepiness and is perhaps the most widely used self-reported sleep tool [55]. However, the ESS measures only propensity to sleep during the day and misses other aspects of sleep. The Pittsburgh Sleep Quality Index (PSQI) includes 19 questions regarding the subjects' sleep pattern over the previous one month that are combined into seven clinically-derived component scores, each weighted equally from 0 to 3. The seven component scores are added to obtain a global score ranging from 0 to 21 . The questions require open-ended answers regarding sleep timing, and multiple-choice responses regarding the severity or frequency of other sleep habits and problems. It has good internal homogeneity, test-retest reliability, discriminant validity [56], and responsiveness to treatments [57] and has been used in the ESRD population $[58,59]$. However, there was limited experience with the Pittsburgh Sleep Quality Index in ESRD patients at the time of the FHN trial design.

The Medical Outcomes Study Sleep Problems Index (SPI) is a 12-item measure that includes items on sleep initiation and maintenance, sleep adequacy, daytime somnolence, and respiratory disturbance; items of the SPI are summed to obtain an overall sleep score. Subjects are instructed to relate responses to sleep habits over the previous month. Because the SPI showed good internal consistency, reliability (Cronbach's $\alpha=0.70$ ) and discriminative validity, with worse sleep quality in HD patients versus patients without known kidney disease [7], the SPI was chosen as the instrument to assess sleep quality in the FHN study.

\section{Self-Perceived Burden on Caregiver}

The Self-Perceived Caregiver Burden Scale is a measure to identify patients' feelings of being a burden on others. The conceptual framework and scale items were derived from qualitative interviews with ESRD patients and health professionals. This HRQOL measure was important given the potential impact on caregivers of more frequent HD. The Self-Perceived Burden Scale was a measure of caregiver burden developed in the ESRD population. These items were then administered in a construct validation to 100 outpatients undergoing $H D$. A shorter 
Table 2. Interim analysis of assessment of PROs in FHN

\begin{tabular}{lrrrr}
\hline & Mean \pm SD & Median & 25th percentile & 75th percentile \\
\hline Timing of survey administration after enrollment, days & $7.9 \pm 11.5$ & 4 & 1 & 8 \\
Average time for completion of the FHN HRQOL battery, min & $36.2 \pm 11.7$ & 33 & 28 & 40 \\
\hline
\end{tabular}

Data from 1,071 interviews among 413 FHN participants (participants are defined as subjects who were enrolled but not necessarily randomized in the trials).

form of the Self-Perceived Burden Scale (10-item abbreviation) was used in the FHN Trials since this comprised much of the variability of the longer form and had adequate internal consistency reliability $(\alpha=0.85)$ [60].

\section{Dialysis-Specific Questions}

There were 8 questions that related to dialysis outcomes including time to recovery after dialysis, adherence to medications, preference for dialysis modality, and three items regarding sexual function. Post-HD fatigue, a common, often incapacitating symptom, has been shown to be less prevalent in daily HD patients compared to conventional thrice-weekly $\mathrm{HD}[25,26,61]$. 'Time to recover from HD session', which measures this aspect has been shown to be a reliable and valid measure of HRQOL and is sensitive to change in the ESRD population [61].

Although the issue of how best to measure medication compliance remains controversial [62], self-reporting has been shown to provide similar estimates of overall adherence as compared to electronic and prescription refill adherence measurement ( $84 \%$ vs. $86 \%$ vs. $91 \%$, respectively) [63]. Medication adherence in the FHN study was assessed by patients' self-reporting of missing doses.

\section{Methods of Administration of HRQOL Tools in FHN}

Self-administered questionnaires may be more difficult to complete for the elderly, minority groups and those with high comorbidity, and may exclude patients with low literacy, visual problems and diminished dexterity creating selection bias [64]. Furthermore, it is not possible to blind patients and their health care providers to the assigned dialysis intervention in the FHN trials. PROs such as HRQOL are considered by some to be subjective in their assessment, and their ascertainment could be influenced by individuals who record survey data if they were knowledgeable about the assigned dialysis intervention and have preconceived notion of its benefit. To address these considerations, the HRQOL questionnaires were centrally administered by trained interviewers using computer-assisted telephone interviewing. HRQOL was assessed at baseline, at 4-5 months, and at 12-14 months after randomization by interviewers blinded to treatment allocation through a central telephone service at the University Center for Social and Urban Research at the University of Pittsburgh. The instruments were completed in the following order: SF-36 Survey, HUI3, BDI, SPI, Caregiver Burden, and additional items including assessment of sexual function and time to recovery.

Once a subject was enrolled in the study, the study coordinator obtained basic demographic information for the HRQOL Central Interviewing Center's Patient Tracking Database. The baseline HRQOL Interview was prior to randomization. If the participant was under the age of 18 years in the Daily Trial, Beck Depression Inventory and the Sexual Function items were excluded from the interview. The participant was contacted and the survey administered by telephone. On average, the surveys were performed within 8 days of being enrolled in the trials. The average time for completion of 1,071 interviews among 413 FHN participants of the FHN HRQOL assessment was 36 min with a $99 \%$ item completion rate (table 2).

\section{Conclusions}

HRQOL is an important outcome for ESRD patients, and a composite of the SF-36 RAND PHC and mortality was thus chosen as a co-primary outcome in the FHN trials. The HRQOL instruments used in the trials represented a balance between being comprehensive, and being a minimum burden for the patient. The study design was strengthened in that the data collection for HRQOL indices was performed by interviewers blinded to the intervention, thus minimizing potential for interviewer bias. Telephone interviews, which minimize selection biases associated with self-report instruments, ensured that 
assessments were conducted in a timely manner, and minimized both general patient-level nonresponse (once recruited) and item nonresponse. Future trials may want to consider using computer-assisted telephone interviews to collect PROs in this patient population. Accurate assessment of HRQOL and PRO can help us determine whether HD interventions improve the health and wellbeing of this vulnerable patient population.

\section{Acknowledgements}

This work was supported by grants from the National Institute of Diabetes and Digestive and Kidney Diseases (U01 DK03005) and Dr. Mark Unruh (DK077785). The FHN investigator list is available in reference [16].

\section{References}

1 Merkus MP, et al: Physical symptoms and quality of life in patients on chronic dialysis: results of The Netherlands Cooperative Study on Adequacy of Dialysis (NECOSAD). Nephrol Dial Transplant 1999;14:1163-1170.

2 Parfrey PS, et al: Clinical features and severity of nonspecific symptoms in dialysis patients. Nephron 1988;50:121-128.

3 Jhamb M, et al: Fatigue in patients receiving maintenance dialysis: a review of definitions, measures, and contributing factors. Am J Kidney Dis 2008;52:353-365.

4 Weisbord SD, et al: Prevalence, severity, and importance of physical and emotional symptoms in chronic hemodialysis patients. J Am Soc Nephrol 2005;16:2487-2494.

5 Agganis BT, et al: Depression and cognitive function in maintenance hemodialysis patients. Am J Kidney Dis 2010;56:704-712.

6 Park HC, et al: Depression and health-related quality of life in maintenance hemodialysis patients. Clin Nephrol 2010;73:374-380.

7 Unruh ML, et al: Sleep quality and clinical correlates in patients on maintenance dialysis. Clin Nephrol 2003;59:280-288.

8 Kimmel PL, Cohen SD, Weisbord SD: Quality of life in patients with end-stage renal disease treated with hemodialysis: survival is not enough! J Nephrol 2008;21(suppl 13):S54-S58.

-9 Ramkumar N, et al: Patient preferences for in-center intense hemodialysis. Hemodial Int 2005;9:281-295.

10 DeOreo PB: Hemodialysis patient-assessed functional health status predicts continued survival, hospitalization, and dialysis-attendance compliance. Am J Kidney Dis 1997;30: 204-212.

11 Lowrie EG, et al: Medical outcomes study short form-36: a consistent and powerful predictor of morbidity and mortality in dialysis patients. Am J Kidney Dis 2003;41: 1286-1292.

12 Mapes DL, et al: Health-related quality of life as a predictor of mortality and hospitalization: the Dialysis Outcomes and Practice Patterns Study (DOPPS). Kidney Int 2003; 64:339-349.
13 DeOreo PB: The use of patient-based instruments to measure, manage, and improve quality of care in dialysis facilities. Adv Ren Replace Ther 2001;8:125-130.

14 Meyer KB, et al: Monitoring dialysis patients' health status. Am J Kidney Dis 1994; 24:267-279.

15 Kimmel PL, et al: Aspects of quality of life in hemodialysis patients. J Am Soc Nephrol 1995;6:1418-1426.

16 Suri RS, et al: Frequent Hemodialysis Network (FHN) randomized trials: study design. Kidney Int 2007;71:349-359.

17 Unruh M, et al: Effects of hemodialysis dose and membrane flux on health-related quality of life in the HEMO Study. Kidney Int 2004;66:355-366.

18 Bayliss G, Danziger J: Nocturnal versus conventional haemodialysis: some current issues. Nephrol Dial Transplant 2009;24: 3612-3617.

19 Suri RS, et al: Daily hemodialysis: a systematic review. Clin J Am Soc Nephrol 2006;1: 33-42.

20 Walsh M, et al: A systematic review of the effect of nocturnal hemodialysis on blood pressure, left ventricular hypertrophy, anemia, mineral metabolism, and health-related quality of life. Kidney Int 2005;67:15001508 .

21 Lockridge RS Jr: Daily dialysis and longterm outcomes - the Lynchburg Nephrology NHHD experience. Nephrol News Issues 1999;13:16, 19, 23-26.

22 McPhatter LL, et al: Nightly home hemodialysis: improvement in nutrition and quality of life. Adv Ren Replace Ther 1999;6:358365.

23 Fong E, Bargman JM, Chan CT: Cross-sectional comparison of quality of life and illness intrusiveness in patients who are treated with nocturnal home hemodialysis versus peritoneal dialysis. Clin J Am Soc Nephrol 2007;2:1195-1200.

24 Manns BJ, et al: Nocturnal hemodialysis does not improve overall measures of quality of life compared to conventional hemodialysis. Kidney Int 2009;75:542-549.
25 Heidenheim AP, et al: Patient quality of life on quotidian hemodialysis. Am J Kidney Dis 2003;42(suppl 1):36-41.

26 Jaber BL, et al: Effect of daily hemodialysis on depressive symptoms and postdialysis recovery time: interim report from the FREEDOM (Following Rehabilitation, Economics and Everyday-Dialysis Outcome Measurements) Study. Am J Kidney Dis 2010;56:531539.

27 Kalantar-Zadeh K, Unruh M: Health related quality of life in patients with chronic kidney disease. Int Urol Nephrol 2005;37:367-378.

28 Unruh ML, Weisbord SD, Kimmel PL: Health-related quality of life in nephrology research and clinical practice. Semin Dial 2005; 18:82-90.

29 Ware J, Keller S: Quality of Life and Pharmacoeconomics in Clinical Trials, ed 2. Interpreting General Health Measures, 1996, pp 445-460.

30 Ware J, et al: SF-36 Health Survey Manual and Interpretation Guide. Boston, The Health Institute, 1993.

31 Bergner M, et al: The Sickness Impact Profile: development and final revision of a health status measure. Med Care 1981;19: 787-805.

32 Campbell A, Converse P, Rogers W: The Quality of American Life: Perceptions, Evaluations and Satisfactions. New York, Russell Sage Foundation, 1976.

33 Hornberger JC, Redelmeier DA, Petersen J: Variability among methods to assess patients' well-being and consequent effect on a cost-effectiveness analysis. J Clin Epidemiol 1992;45:505-512.

34 Hunt S, McEwan J, McKenna SP: Measuring Health Status. London, Croom Helm, 1986.

35 Bergner M: Development, testing and use of the Sickness Impact Profile Quality of Life Assessment: Key Issues in the 1990s. Dordrecht, Kluwer Academic Publishers, 1993.

36 Edgell ET, et al: A review of health-related quality-of-life measures used in end-stage renal disease. Clin Ther 1996;18:887-938. 
-37 Cagney KA, et al: Formal literature review of quality-of-life instruments used in end-stage renal disease. Am J Kidney Dis 2000;36:327336.

38 Kurtin PS, et al: Patient-based health status measures in outpatient dialysis. Early experiences in developing an outcomes assessment program. Med Care 1992;30(suppl): MS136-MS149.

-39 Rettig RA, Sadler JH: Measuring and improving the health status of end stage renal disease patients. Health Care Financ Rev 1997; 18:77-82.

40 Beusterien KM, et al: The effects of recombinant human erythropoietin on functional health and well-being in chronic dialysis patients. J Am Soc Nephrol 1996;7:763-773.

41 Nortvedt MW, et al: Performance of the SF36, SF-12, and RAND-36 summary scales in a multiple sclerosis population. Med Care 2000;38:1022-1028.

42 Churchill DN, et al: Measurement of quality of life in end-stage renal disease: the time trade-off approach. Clin Invest Med 1987;10: 14-20.

43 Laupacis A, Wong C, Churchill D: The use of generic and specific quality-of-life measures in hemodialysis patients treated with erythropoietin. The Canadian Erythropoietin Study Group. Control Clin Trials 1991; 12(suppl):168S-179S

44 Liem YS, Bosch JL, Hunink MG: Preferencebased quality of life of patients on renal replacement therapy: a systematic review and meta-analysis. Value Health 2008;11:733741.

45 Torrance GW: Preferences for health outcomes and cost-utility analysis. Am J Manag Care 1997;(suppl):S8-S20.
46 Horsman J, et al: The Health Utilities Index (HUI): concepts, measurement properties and applications. Health Qual Life Outcomes 2003;1:54.

-47 Baldassarre FG, et al: Effect of coronary artery bypass graft surgery on older women's health-related quality of life. Heart Lung 2002;31:421-431.

48 Schunemann HJ, et al: Evaluation of the minimal important difference for the feeling thermometer and the St. George's Respiratory Questionnaire in patients with chronic airflow obstruction. J Clin Epidemiol 2003; 56:1170-1176.

49 Cohen SD, et al: Screening, diagnosis, and treatment of depression in patients with endstage renal disease. Clin J Am Soc Nephrol 2007;2:1332-1342.

50 Hedayati SS, Finkelstein FO: Epidemiology, diagnosis, and management of depression in patients with CKD. Am J Kidney Dis 2009; 54:741-752.

51 Shidler NR, Peterson RA, Kimmel PL: Quality of life and psychosocial relationships in patients with chronic renal insufficiency. Am J Kidney Dis 1998;32:557-566.

52 Kimmel PL: Psychosocial factors in adult end-stage renal disease patients treated with hemodialysis: correlates and outcomes. Am J Kidney Dis 2000;35(suppl 1):S132-S140.

53 Kimmel PL, et al: Psychiatric illness in patients with end-stage renal disease. Am J Med 1998;105:214-221.

54 Moul DE, et al: Self-report measures of insomnia in adults: rationales, choices, and needs. Sleep Med Rev 2004;8:177-198.

55 Johns MW: A new method for measuring daytime sleepiness: the Epworth sleepiness scale. Sleep 1991;14:540-545.

56 Buysse DJ, et al: The Pittsburgh Sleep Quality Index: a new instrument for psychiatric practice and research. Psychiatry Res 1989; 28:193-213.
57 King AC, et al: Moderate-intensity exercise and self-rated quality of sleep in older adults. A randomized controlled trial. JAMA 1997; 277:32-37.

58 Carpenter JS, Andrykowski MA: Psychometric evaluation of the Pittsburgh Sleep Quality Index. J Psychosom Res 1998;45:513.

59 Iliescu EA, et al: Quality of sleep and healthrelated quality of life in haemodialysis patients. Nephrol Dial Transplant 2003;18: 126-132.

60 Cousineau N, et al: Measuring chronic patients' feelings of being a burden to their caregivers: development and preliminary validation of a scale. Med Care 2003;41:110 118.

61 Lindsay RM, et al: Minutes to recovery after a hemodialysis session: a simple health-related quality of life question that is reliable, valid, and sensitive to change. Clin J Am Soc Nephrol 2006;1:952-959.

62 Chang TI, Winkelmayer WC: Kidney disease and antihypertensive medication adherence: the need for improved measurement tools. Am J Kidney Dis 2010;56:423-426.

63 Hansen RA, et al: Comparison of methods to assess medication adherence and classify nonadherence. Ann Pharmacother 2009;43: 413-422.

64 Unruh M, et al: Bias in assessment of healthrelated quality of life in a hemodialysis population: a comparison of self-administered and interviewer-administered surveys in the HEMO study. J Am Soc Nephrol 2003;14: 2132-2141. 